\title{
EXPANDING RELATIVISTIC SHELLS AND GAMMA-RAY BURST TEMPORAL STRUCTURE
}

\author{
Edward E. Fenimore, ${ }^{1}$ Claudine D. Madras, and Sergei Nayakshin \\ D436, Los Alamos National Laboratory, Los Alamos, NM 87545 \\ Received 1996 January 29 ; accepted 1996 July 11
}

\begin{abstract}
Many models of gamma-ray bursts (GRBs) involve a shell expanding at extreme relativistic speeds. The shell of material expands in a photon-quiet phase for a period $t_{0}$ and then becomes gamma-ray active, perhaps due to inhomogeneities in the interstellar medium or the generation of shocks. Based on kinematics, we relate the envelope of the emission of the event to the characteristics of the photon-quiet and photon-active phases. We initially assume local spherical symmetry wherein, on average, the same conditions prevail over the shell's surface within angles the order of $\Gamma^{-1}$, where $\Gamma$ is the Lorentz factor for the bulk motion. The contribution of the curvature to the temporal structure is comparable to the contribution from the overall expansion. As a result, GRB time histories from a shell should have an envelope similar to "FRED" (fast rise, exponential decay) events in which the rise time is related to the duration of the photon-active phase and the fall time is related to the duration of the photon-quiet phase. This result depends only on local spherical symmetry and, since most GRBs do not have such envelopes, we introduce the "shell symmetry" problem: the observed time history envelopes of most GRBs do not agree with that expected for a relativistic expanding shell.

Although FREDs have the signature of a relativistic shell, they may not be due to a single shell, as required by some cosmological models. Some FREDs have precursors in which the peaks are separated by more than the expansion time required to explain FRED shape. Such a burst is most likely explained by a central engine; that is, the separation of the multiple peaks occurs because the central site produced multiple releases of energy on timescales comparable to the duration of the event. Alternatively, there still could be local spherical symmetry of the bulk material, but with a low "filling factor"; that is, only a few percent of the viewable surface (which is already very small, $4 \pi \Gamma^{-2}$ ) ever becomes gamma-ray active.

Long complex bursts present a myriad of problems for the models. The duration of the event at the detector is $\sim t_{0} /\left(2 \Gamma^{2}\right)$. The long duration cannot be due to large $t_{0}$, since it requires too much energy to sweep up the interstellar medium. Nor can it be due to small $\Gamma$ if the time variation is due to ambient objects, since the density of such objects is unreasonable $\left(\sim 10^{18} \Gamma^{-4} \mathrm{pc}^{-3}\right.$ for typical parameters $)$. Long events must explain why they almost always violate local spherical symmetry or why they have low filling factors.

Both precursor and long complex events are likely to be "central engines" that produce multiple releases of energy over $\sim 100 \mathrm{~s}$. One promising alternative scenario is one in which the shell becomes thicker than the radius of the curvature within $\Gamma^{-1}$. Then it acts as a parallel slab, eliminating the problems associated with local spherical symmetry.
\end{abstract}

Subject headings: gamma rays: bursts - radiative transfer

\section{INTRODUCTION}

Although gamma-ray bursts (GRBs) have been observed and studied for nearly 25 years, little has been concluded about the source of the bursts. In particular, their distance could be either cosmological (Paczyński 1995) or galactic (Lamb 1995). The presence of photons well above the pair production threshold (e.g., $18 \mathrm{GeV}$; Hurley et al. 1995) has deepened the mystery; only extreme relativistic motion will allow the escape of such radiation. The predicted amount of relativistic motion depends on estimates of the distance to and the size of the source. The accepted method of estimating the size is to use the temporal variations in the time histories together with causality arguments to set an upper limit. Originally Schmidt (1978) estimated the Lorentz $\Gamma$ factor based on the lack of observed photon-photon attenuation and the causality argument that the size of an object is limited to $c \Delta T_{p}$, where the time $\Delta T_{p}$ is the duration of a peak within a burst $\left[\right.$ Here the Lorentz $\Gamma$ is $\left(1-\beta^{2}\right)^{-1 / 2}$, where

\footnotetext{
${ }^{1}$ efenimore@lanl.gov.
}

$\beta=v / c$ and $v$ is the bulk speed of radiating particles.] Using $c \Delta T_{p}$ assumes a static location for the emitting surface.

When it became clear that GRBs could be at cosmological distances (see Meegan et al. 1992), the resulting energy release $\left(10^{51} \mathrm{ergs} \mathrm{s}^{-1}\right)$ implied a relativistic expanding shell. Fenimore et al. (1992) and Fenimore, Epstein, \& Ho (1993a) estimated the Lorentz $\Gamma$ factor based on a relativistic expanding shell with a size of $\sim 2 \Gamma^{2} c \Delta T_{p}$. This size implies that the central site acts as a "central engine" for the burst, ejecting material in a fluctuating series of shells that cause the peaks within the burst. Mészáros \& Rees (1992) suggested that there is only a single release of energy at the central site resulting in one shell. In these models, a single shell of expanding material becomes the source of gamma rays. In this paper, we investigate ways that fluctuations in the time history can be related to the size of the emitting surface of a relativistic expanding shell. We will base our restrictions on kinematic considerations. This complements analyses based on hydrodynamic timescales (see, e.g., Mészáros \& Rees 1993; Sari \& Piran 1995). 

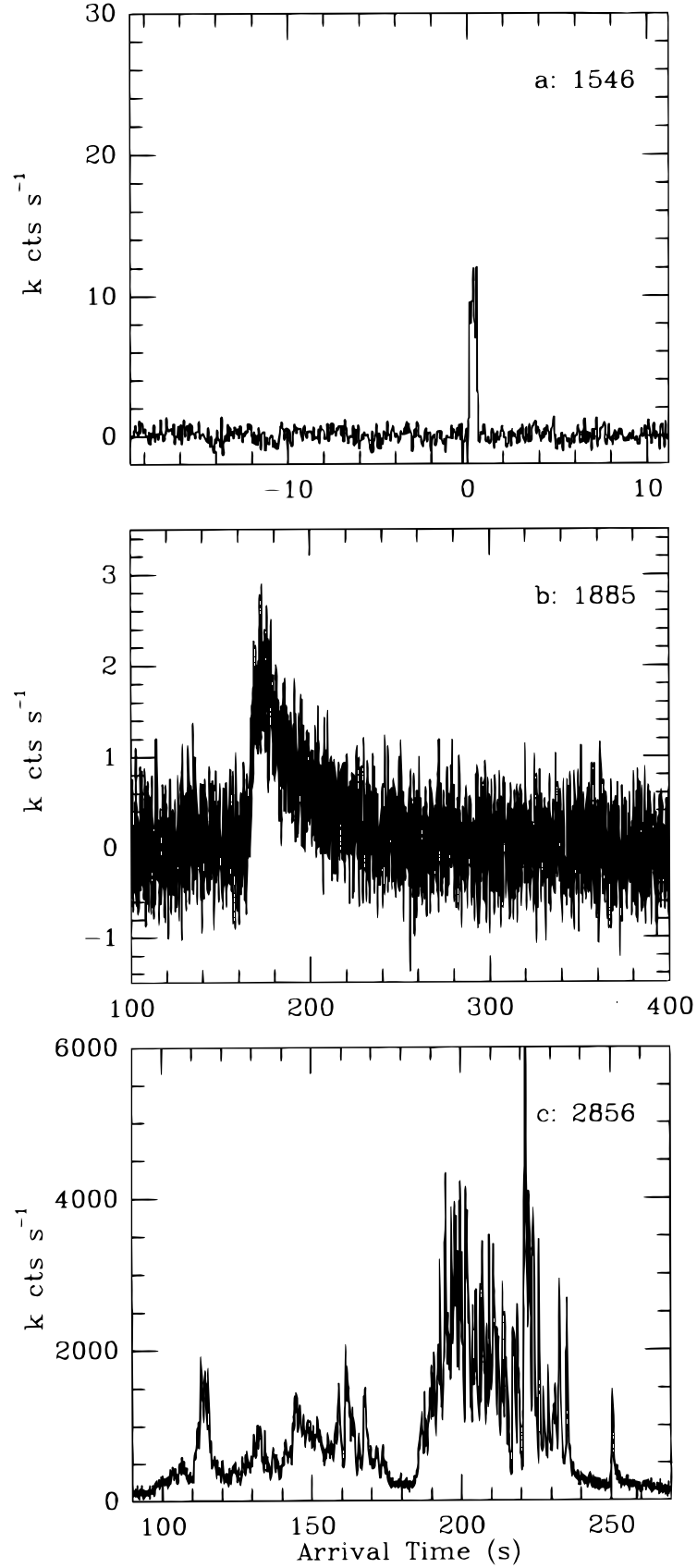

FIG. 1.-The diversity of GRB time histories. (a) Burst 1546 is a sharp single spike with a similar rise and fall time. (b) Burst 1885 is a FRED (fast rise, exponential decay). A relativistic shell can appear to the observer as a FRED, although the decay phase is actually a power law rather than exponential. (c) Burst 2856 is a long complex burst. Note that the spikes are about the same size at the beginning as at the end.

A superrelativistic, expanding shell is a common scenario for cosmological models, since many energy release mechanisms occur at the dynamic timescale for compact objects (a small fraction of a second) (Piran, Shemi, \& Narayan 1993; Katz 1994; Mészáros \& Rees 1993; Shemi 1994; Piran \& Shemi 1993) or over a few seconds (Mészáros \& Rees 1994; Rees \& Mészáros 1994). However, GRBs often display chaotic time histories that last many seconds and have rapid variations on timescales that are small compared to the duration of the event. Thus, although the energy released at the central site produces material that expands steadily outward, the photon production is not constant. Figure 1 shows the diversity of GRB time histories observed by the Burst And Transiant Source Experiment (BATSE). Many GRBs have somewhat simple temporal structure, either single spikes such as in Figure $1 a$ or a fast rise, exponential decay (or FRED; see Fig. 1b). Other bursts show a series of complex peaks, as in Figure 1c. From each burst, one can estimate a timescale of variation $\Delta T_{p}$, at each point $T_{p}$ within the burst. For example, the complicated burst 2856 shows numerous peaks with $\Delta T_{p}$ of $\sim 1$ s for about 150 $\mathrm{s}$ (see Fig. 1c). We denote the time of onset of a peak as $T_{p}$. Although statistics or temporal resolution might hide the true number of peaks in burst 2856, detailed fitting with a generalized pulse shape demonstrates that GRB pulse width average $\sim 0.6 \mathrm{~s}$, so most of the individual peaks seen in bursts are probably distinct entities (Norris et al. 1996).

Clearly, the rate of photon production is not related directly to either the short timescale of the energy release ( $\lesssim \mathrm{a}$ few seconds) or the area of an expanding shell; the time histories do not follow an envelope that scales as $T_{p}^{2}$. Rather, the photon production within the expanding shell must vary due to other reasons. For example, the expanding shell might run into the interstellar medium (ISM), resulting in temporal variations (Mészáros \& Rees 1993). Other models hypothesize that the gamma rays result from ambient photons that are up scattered by the relativistic particles. The temporal variations could be due to the variations in the ambient photon density that might be found near an active galactic nucleus (AGN) (Epstein et al. 1993) or a collapsed core of a globular cluster (Shemi 1994; Shaviv \& Dar 1995). Alternatively, the growth and decay of relativistic shocks within the shell could produce the observed rapid time variations (Mészáros \& Rees 1994).

In this paper, we seek to explain the overall envelope of GRB time histories. Given the variety of models that have been suggested, we must cover many possibilities. The reader needs to be aware that, since this paper addresses the wide range of suggested explanations for the time structure, different (or even conflicting) assumptions will be made in different parts of the paper. In $\S 2$ we develop the general technique for relating emission of the shell to the observations. In $\S 3$ we present five different scenarios (with different underlying assumptions) that could explain some substructure within the bursts and/or the overall duration of events. In $\S 4$ we analyze particular types of GRB time histories in terms of the scenarios discussed in $\S 3$. In general, it is not possible to explain the diversity of GRB time histories with a single set of consistent assumptions.

\section{EFFECTS OF DURATION AND THICKNESS ON TIME STRUCTURE}

Consider a single, relativistic, expanding shell characterized by the Lorentz factor $\Gamma$ associated with the bulk motion. We will assume that all motion is radial and that the shell is spherically symmetric in the frame of the explosion. In fact, the symmetry of expansion matters only over angles the order of a few times $\Gamma^{-1}$; beaming prevents us from observing other regions of the shell. Furthermore, the relatively large Lorentz factor associated with GRBs causes important differences with other situations involving relativistic motion such as AGNs. Consider the case in which the shell is not spherically symmetric but rather is a jet of angular width $\theta_{B}$ (as assumed by several authors, e.g., Krolik \& Pier 1991). When the Lorentz factor is small, it is 
likely that an observer will see an edge of a jet rather than be positioned directly within $\theta_{B}$ and see the jet head on. The opposite is true when the Lorentz factor is very large. The chances that one sees the side of the jet is the probability that the angle of the observer line of sight is larger than $\theta_{B}$ but smaller than $\theta_{B}+\Gamma^{-1}$. This probability is about $\left(\theta_{B} \Gamma\right)^{-1}$. Given the large energy release $\left(\sim 10^{50} \mathrm{ergs}^{-1}\right)$ and large size $\left(\gg 10^{15} \mathrm{~cm}\right)$, it seems unlikely that there is a mechanism that can routinely confine $\theta_{B}$ to pencil beams much smaller than $\Gamma^{-1}$. When the Lorentz factor is large, an observer either views the material head on or probably will not see the source at all. One cannot treat GRBs with large Lorentz factors as the edges of a jet. Thus, we assume "local spherical symmetry" in which the outflowing material is essentially symmetric about our line of sight on angular scales of $\sim \Gamma^{-1}$.

It is important to recognize that time variability of photon emission can be characterized in three different ways, leading to three different timescales. First, one can measure time in the detector's (or "laboratory") rest frame (DRF). We will ignore any potential motion of the explosion site in our rest frame such as the motion due to the expansion of the universe; these effects are small compared to the effects due to the bulk motion. Thus, the DRF is the same as the rest frame of the explosion. The DRF quantities are denoted without a prime. For example, $t$ is the time in the DRF as measured from the initial release of energy at the central site. Second, one can measure the "proper" time in the comoving frame (CMF) of the shell. We denote quantities measured in the CMF with a prime (e.g., $\left.t^{\prime}\right)$. The quantities in the DRF and CMF are related to each other through a Lorentz transformation. The third timescale concerns how time is measured in the DRF. The rest-frame time, $t$, is determined by clocks placed at each point within the frame, and therefore it is impossible to measure in practice. Rather, a detector is placed at one point to observe temporal variations. This third time we refer to as the "arrival" time (denoted by capital letters, e.g., $T$ ). In practice, the arrival time, the time as in Figure 1, is the only timescale observed. The arrival time, $T$, is related to the DRF time as $T=(1-\beta \cos \theta) t \approx t /\left(2 \Gamma^{2}\right)$ (if there is a single expanding shell). Here $\theta$ is the angle of the motion of the emitting region with respect to the direction to the observer. Thus, when one states that GRBs last tens of seconds and have microsecond time variations, one is actually referring to arrival time, not the time in the CMF or the DRF. The initial explosion is at time $T=t=0$ and forms a shell that expands as $r=v t$. Since in most models the gamma-ray-emitting phase does not start at the time of the initial explosion, one does not know where to place $T=0$. At radius $r_{0}=v t_{0}$, the shell begins to emit gamma rays. These initial photons arrive at the detector at a time we denote at $T_{0}$. If $r_{0}$ is large, $t_{0}$ cannot be neglected in calculations. In $\S 4$, we discuss how we use the results of this paper to assign when the initial explosion might have occured.

Note that we define all times, $T, t$, and $t^{\prime}$, to be measured from the initial energy release, so that a peak arriving, say, $30 \mathrm{~s}$ after the first gamma rays occurs at time $T_{p}=$ $T_{0}+30 \mathrm{~s}$.

\subsection{Timescales from Thickness and Duration}

An observer viewing a spherically symmetric, relativistically expanding shell from the vantage point of a single position "sees" a shape defined by the photons that arrive at the observer at the same time. These photons originate from a prolate ellipsoid (Rees 1966). After arrival time $T$, the semimajor axis is $\Gamma^{2} v T$, the semiminor axis is $\Gamma v T$, and the eccentricity is $\beta$. In the rest frame of the detector, the distance from the center of the explosion to the point closest to the detector is $v(1+\beta) \Gamma^{2} T \approx 2 \Gamma^{2} c T$ (see Fig. 2). The curvature within $\Gamma^{-1}$ is small, and previous papers

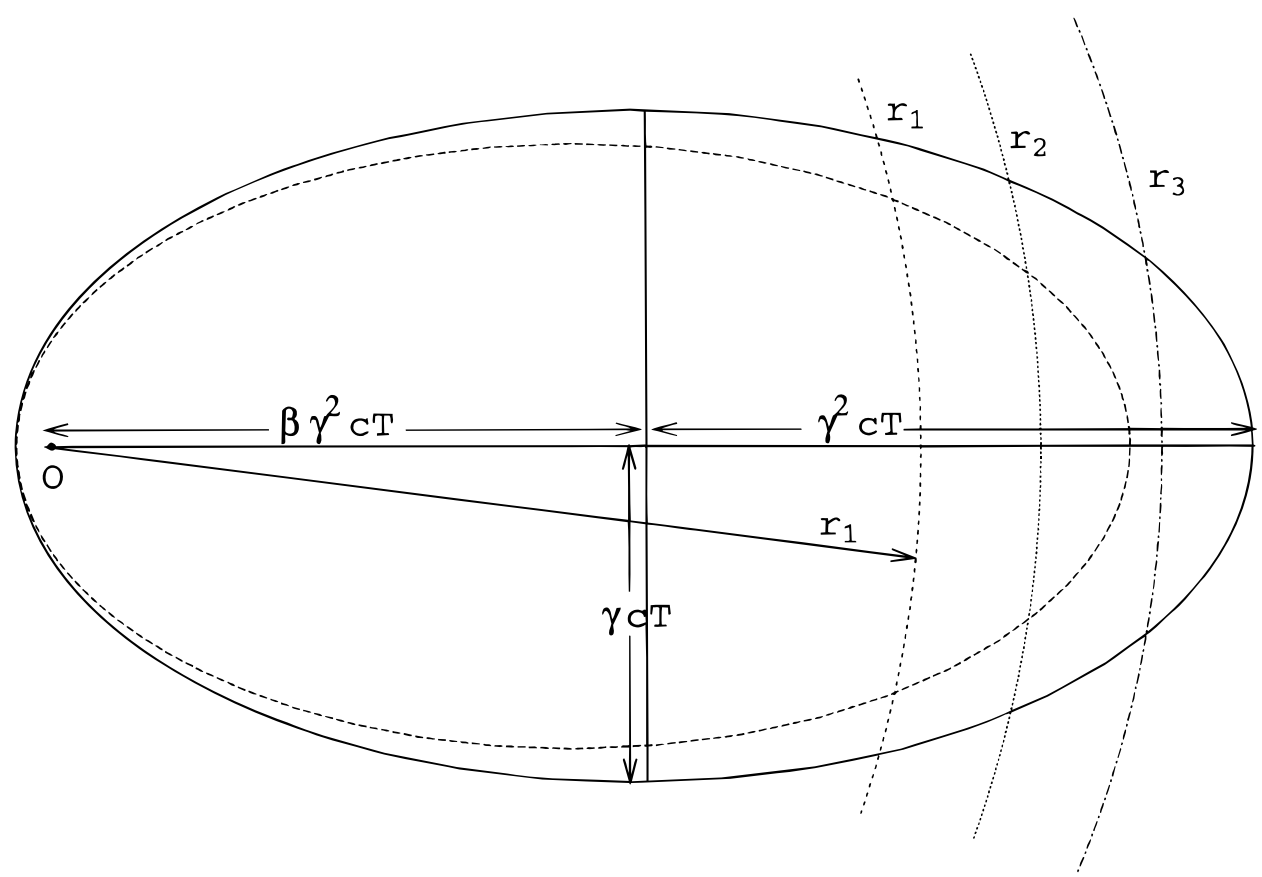

FIG. 2.- The surface that produces photons from a relativistically expanding shell as seen simultaneously by a distant observer. The observer is located at right at infinity. The shell originated at point $O$. Dotted lines at $r_{1}, r_{2}$, and $r^{3}$ represent hypothetical distances at which shells could produce photons. $\Gamma$ is the Lorentz gamma factor of the expansion, and $T$ is the photon arrival time in the detector counted from the moment of the beginning of the explosion. 
have neglected it. However, we will show that the curvature has temporal effects that are comparable to the temporal effects of the overall expansion. To understand this, one must distinguish temporal effects associated with the duration of the emission in the DRF from those effects associated with the thickness of the emitting region in the DRF.

Consider a shell with thickness $\Delta \boldsymbol{r}_{\|}$(as measured in the DRF) that emits for a duration $\Delta t$ from time $t_{1}$ to $t_{2}$ (see Fig. 3). At time $t_{1}$, photon " $\mathrm{A}$ " is emitted from the leading edge of a shell with finite thickness, and photon " $B$ " is emitted from the trailing edge. At time $t_{2}$, photons "a" and " $b$ " are emitted from the leading and trailing edge, respectively. Since the edges are moving at speed $v$ (very close to $c$ ), the photons emitted from the same edge of the shell but at different times (i.e., "A" and "a") arrive at the detector separated in time by $(1-\beta)\left(t_{2}-t_{1}\right) \approx\left(2 \Gamma^{2}\right)^{-1} \Delta t=$ $(2 \Gamma)^{-1} \Delta t^{\prime}$. Thus, the emission from single shells appears to be compressed in arrival time to be $2 \Gamma^{2}$ times shorter than in the DRF and $2 \Gamma$ times shorter than in the CMF.

In contrast, a thick shell emits photons simultaneously (in the DRF) from opposite edges. (e.g., photons " $A$ " and "B" in Fig. 3) which arrive at the detector separated in time by $\Delta r_{\|} / c$. Thus, the emitting region of a GRB with $\sim 1 \mathrm{~s}$ peaks must not have been thicker than 1 light-second in the DRF. One can conclude that at no time throughout either burst in Figures $1 a$ or $1 c$ was the thickness of the emitting region greater than approximately a light-second. We draw this conclusion in general for GRBs; at no time can the regions emitting photons have a line-of-sight width greater than the observed width of the peaks. Since the expanding shell is $\sim 2 \Gamma^{2} T_{0}$ in size, the width of the peaks requires the emitting region to be very thin: $\Delta r_{\|} / r_{0} \sim \Delta T_{0}\left(2 \Gamma^{2} T_{0}\right)^{-1}$, which is about $10^{-2} \Gamma^{-2}$ when $T_{0}$ is as large as hundreds of seconds.

Duration of emission, therefore, causes a peak to appear to be $\left(2 \Gamma^{2}\right)^{-1}$ times shorter in arrival time as it is in the DRF, while thickness of the emitting region causes a peak that appears to the detector as it was emitted in the rest frame. For this reason, even a small thickness in the emitting region can have a great effect on the observed time histories. The delay due to the curvature is a thickness effect $\left(d_{c}\right.$ in Fig. 3). The curvature is $\sim \Gamma^{-2}$ times smaller than the

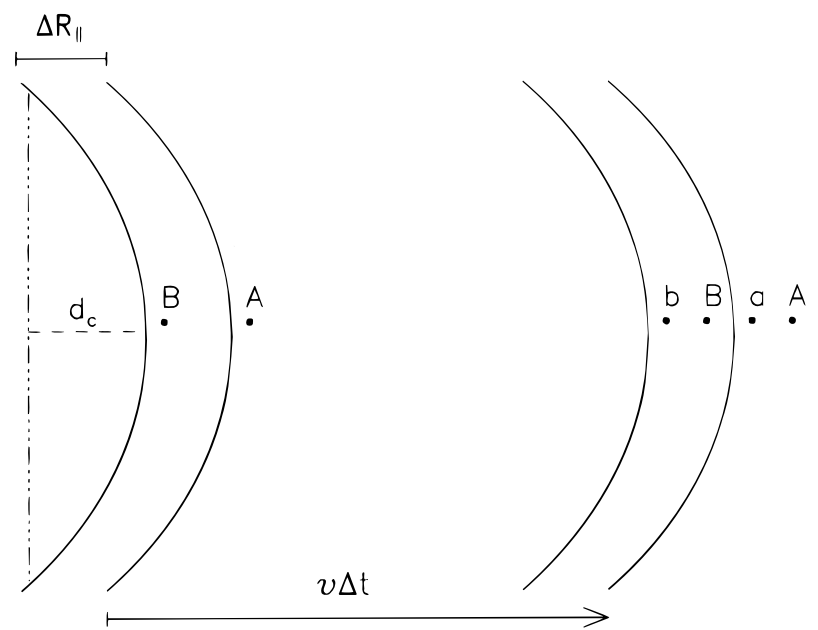

FIG. 3.-The distinction between duration and thickness. A shell with thickness $\Delta r_{\|}$emits photons "A" and "B." After time $\Delta t$, photons " a" and " $b$ " are emitted. The thickness effects are determined by photons " $A$ " and "B," whereas duration effects are determined by photons "A" and "a." Duration effects are contracted by $\Gamma^{2}$, whereas thickness effects are not. Thus, the delay due to the curvature $\left(d_{c}\right)$ is not contracted. radius, but thickness effects are not compressed in time as duration effects are. The expansion is a duration effect and is compressed by $2 \Gamma^{-2}$. Thus, the curvature has an impact comparable to the expansion.

\subsection{Special Case: An Infinitely Thin Shell}

The simplest scenario that demonstrates the effect of the curvature is an infinitely thin shell that expands in a photon-quiet stage and then emits photons for some time $t_{b}$ in the DRF, where $t_{b}$ is the duration of the gamma-active phase. It is clear from Figure 1 that the shell of material does not emit constantly, especially for complex bursts. To describe this analytically, let us define a photon production rate on the shell that varies as position and time. Let $P(\theta, \phi$, $t) d t$ be the number fluence (in units of photons $\mathrm{cm}^{-2}$ ) emitted at location $\theta, \phi$ between time $t$ and $t+d t$. The general expression for the flux (in units of photons $\mathrm{cm}^{-2}$ $\mathrm{s}^{-1}$ ) in the bandpass $E_{1}$ to $E_{2}$ observed between $T$ and $T+d t$ is

$$
V(T)=\frac{1}{D^{2}} \oint_{\text {ellip }} \int_{E_{1} \Lambda}^{E_{2} \Lambda} \frac{\Phi\left(E^{\prime}\right)}{\Delta} P(\theta, \phi, t) \Lambda^{-2} d E^{\prime} d A,
$$

where

$$
\Lambda=\Gamma(1-\beta \cos \theta),
$$

$D$ is the distance to the observer, and the surface differential, $d A$, lies on the surface of the prolate ellipsoid that produces photons that arrive at time $T=t /\left(2 \Gamma^{2}\right)$. The spectrum of the emission in the comoving frame of the shell is $\Phi\left(E^{\prime}\right)$. The $\Lambda^{-1}$ term accounts for the beaming that occurs when one transforms from the rest frame of the expanding shell to the rest frame of the detector. Let $\Lambda_{0}=\Gamma(1-\beta)$, then the factor $\Lambda / \Lambda_{0}$ gives the relative boost of the rest-frame spectrum between those photons that arrive first (i.e., $\Lambda_{0}$ ) and at time $T$ (i.e., $\Lambda$ ). We assume that the emission in the comoving frame of the shell is isotropic.

We try first to make the most narrow time structure possible by assuming that an infinitely thin shell becomes uniformly photon active for an infinitesimal time $d t$, i.e., $P(\theta, \phi$, $t)=P_{0} \delta\left(t-t_{0}\right)$. Previously, we assumed local spherical symmetry for the outflowing material. Here, by assuming that $P(\theta, \phi, t)$ is independent of $\theta, \phi)$, we are adding the additional assumption of local spherical symmetry for the conversion of bulk energy into gamma rays. We will relax this assumption later. The distance from the initial explosion to a point on the ellipsoid defined by the angle $\theta, r_{\mathrm{el}}$, is $r_{\mathrm{el}}=v t(1-\beta \cos \theta)^{-1}$. The surface that corresponds to the $\delta$-function emission is characterized in arrival time as

$$
r_{0}=\frac{v T_{0}}{1-\beta}=\frac{v T}{1-\beta \cos \theta},
$$

such that

$$
\cos \theta=\frac{1-\left(T / T_{0}\right)(1-\beta)}{\beta},
$$

and

$$
\Lambda=\frac{T / T_{0}}{(1+\beta) \Gamma} .
$$

The $d A$ in equation (1) is the same as the $d A$ of a spherical shell facing the observer; that is,

$$
d A=2 \pi r_{0}^{2} \sin \theta \cos \theta d \theta .
$$


Using equation (3),

$$
d A=\frac{\pi r_{0}^{2}}{\Gamma^{2} T_{0}} d T=4 \pi \Gamma^{2} c^{2} T_{0} d T .
$$

The resulting time variation for a single shell emitting for a short time period, as seen by the observer, is

$$
V_{\delta}(T) d T= \begin{cases}0 & \text { if } T<T_{0}, \\ \psi \Gamma^{4+\alpha} P_{0}\left(\frac{T}{T_{0}}\right)^{-\alpha-2} T_{0} d T & \text { if } T>T_{0},\end{cases}
$$

where we have assumed that the rest-frame photon number spectrum is a power law with index $-\alpha$, and $\psi$ is the constant,

$$
\psi=\frac{2^{4+\alpha} c^{2} \pi}{D^{2}} \int_{E_{1}}^{E_{2}} E^{-\alpha} d E
$$

Since the emission is assumed to occur at a single radius, changes in $\Gamma$ (such as slowing down in the ISM) do not affect the shape.

As long as $\theta \sim \Gamma^{-1}$ is small, the time variation in $V_{\delta}(T)$ depends only on $T / T_{0}$; the shape is shown in Figure $4 a$ for $\alpha=1.5$. Note that the shape depends on where $T$ is defined to be zero, which is when the central explosion occurred. The FWHM of $V_{\delta}(T)$ varies from $0.26 T_{0}$ to $0.19 T_{0}$ as $\alpha$ varies from 1 to 2 . Thus, the shape expected for a single shell that turns on is rather universal: it has a weak dependency on the spectral shape. For the purposes of examples in this paper, we will use $\alpha=1.5$ such that the FWHM of $V_{\delta}(T) \sim$ $0.22 T_{0}$. This shape is similar to the time histories of the GRBs referred to as FREDs, which are characterized by their fast rise times and long tails. We propose that FREDs result from an expanding shell that emits from a small range of times at $t_{0}$. Although called "FREDs," according to equation (8) the tail is not exponential but $T^{-\alpha-2}$. If all bursts were FRED-like, then relativistic shells that turn on after a time $T_{0}=t_{0}\left(2 \Gamma^{2}\right)^{-1}$ would explain easily the envelope of emission.

\subsection{Infinitely Thin Shells Emitting for Finite Time}

The shape $V_{\delta}(T)$ was derived assuming that $P(\theta, \phi, t)$ was a $\delta$-function. More complex envelopes can be found as weighted sums of $V_{\delta}(T)$. For example, as the bulk material converts its energy to gamma rays, $\Gamma$ should decrease. We assume that $\Gamma(T)$ can be approximated as $\Gamma_{0}\left(T_{e} / T_{0}\right)^{-\zeta}$, where $T_{e}$ is the time of emission. If $P(\theta, \phi, t)$ has local spherical symmetry $[=P(T)]$ and is constant $\left(=P_{0}\right)$ from $t=T_{0} 2 \Gamma^{2}$ to $t=T_{\max } 2 \Gamma^{2}$ and zero otherwise, then

$$
V(T)=\psi \int_{T_{0}}^{T} \Gamma^{4+\alpha}\left(T_{e}\right)\left(\frac{T}{T_{e}}\right)^{-\alpha-2} P\left(T_{e}\right) T_{e} d T_{e},
$$

such that

$$
V(T)= \begin{cases}0 & \text { if } T<T_{0}, \\ \frac{\psi \Gamma_{0}^{4+\alpha} P_{0}}{\omega} \frac{T^{\omega}-T_{0}^{\omega}}{T_{0}^{-4 \zeta} T^{\alpha+2}} & \text { if } T_{0}<T<T_{\max }, \\ \frac{\psi \Gamma_{0}^{4+\alpha} P_{0}}{\omega} \frac{T_{\max }^{\omega}-T_{0}^{\omega}}{T_{0}^{-4 \zeta} T^{\alpha+2}} & \text { if } T>T_{\max },\end{cases}
$$

where

$$
\omega=\alpha-(4+\alpha) \zeta+4 .
$$
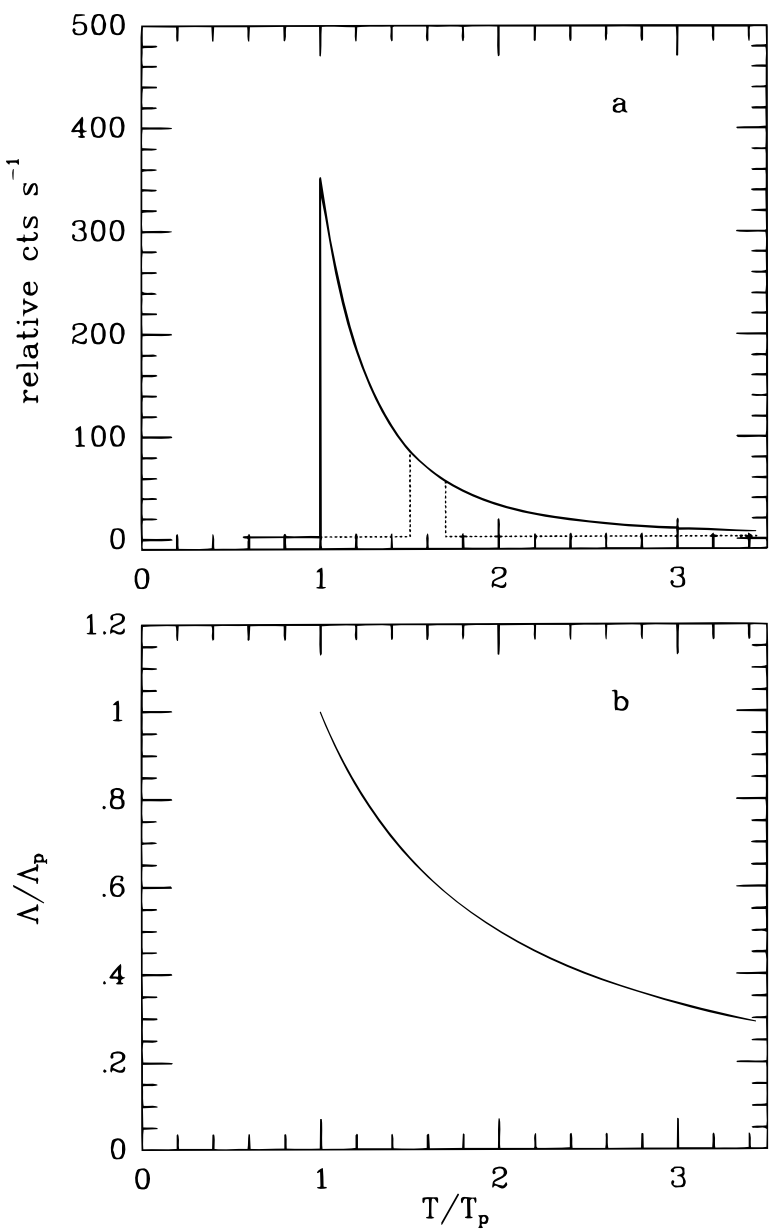

FIG. 4.- Time history expected from a relativistic shell that generates gamma rays after expanding in a gamma-quiet phase for time $T_{0}$. (a) The shape is a fast rise with a power-law decay. The width of the time structure is proportional to the time spent in the gamma-quiet phase. Dotted lines show a possible time history if only a single small patch on the shell becomes active. (b) Relative boosting of the photons from the frame comoving with the shell. Photons at the peak of the time history originate from the portion of the shell that is closest to the observer. Photons from the decay phase originate off axis, and the Lorentz boost is smaller by $T / T_{0}$.

If $\Gamma$ decreases to $\Gamma_{\min }$, then

$$
\zeta \sim \ln \left(\Gamma_{0} / \Gamma_{\min }\right) / \ln \left(T_{\max } / T_{0}\right),
$$

where $\Gamma_{\min }$ is the $\Gamma$ at $T_{\max }$. Below we argue that $\Gamma$ cannot vary much because the observed peaks are often about the same width at the end of the burst as at the beginning. Thus, $\zeta$ is probably small. For small $T_{0}$, this envelope is a pulse with a rise that follows $\sim T^{2-4(\alpha+\zeta)}$ and a fall that follows $T^{-\alpha-2}$. Other functions for $P(T)$ can give sharper rises. For example, if the shell runs into some material such that $P(T)$ is a linearly increasing function from $T_{0}$ to $T_{\max }$, then the pulse can rise as $\sim T^{3-4(\alpha+\zeta)}$. If the falling density cause $P(T)$ to decrease with $T$, then the pulse will rise slower than $T^{2-4(\alpha+\zeta)}$

\subsection{Infinitely Thin Shells Emitting at Multiple Radii}

Many bursts do not have a simple FRED-like shape but consist of many peaks. One could have emission at multiple radii; that is, have the shell emit at multiple times $\left(T_{p}\right)$. To investigate multiple radii, one can add functions like equation (8) with $T_{0}$ replaced with $T_{p}$. A visual inspection of the BATSE catalog of multiple-peaked time histories 
reveals that usually peaks have about the same duration at the beginning of the burst as near the end of the burst; that is, $\Delta T_{p}$ is roughly constant for a burst (see e.g., Fig. $1 c$ ). Equation (8) predicts that peaks should become progressively wider (i.e., the FWHM is $\alpha T_{p}$ and $T_{p}=T_{0}$ plus time from the first gamma-ray peak). If peaks all originate with a large $T_{0}$, the amount that the peaks widen will be smaller, but then each peak must already be wide. This is not observed (see discussion of burst 219 in $\S 4$ ). Note that $T / T_{p}=t / t_{p}$, so the value of $\Gamma$ does not affect how the peaks become progressively wider.

\section{RELATING PHYSICAL SIZE TO TEMPORAL STRUCTURE}

Except for FREDs, single infinity thin shells do not predict complex time histories (see $\S 2$ ). In this section, we lift the assumption that the conversion of the bulk motion into gamma rays is locally spherically symmetric. The thickness and the angular extent of the resulting emitting regions as well as the duration of emission all contribute to form the time histories. We will now look at five different scenarios and how the observed time structure (e.g., pulse widths and the burst duration) can be related to the physical size of the emitting region.

\subsection{Simultaneous Conditions}

A shell expanding outward could become photon active, turning on and off because the appropriate conditions for photon production occur at roughly the same time. Such regions might occur in models of a shell sweeping up the ISM or internal shock growth. In this case, causality considerations do not limit the size of the regions; a shell sweeping forward may become photon active at the same time in regions whose size is not limited by $c t^{\prime}$. Let $\Delta r_{\perp}$ be the length of the arc that becomes photon active. Assuming for simplicity that the active region is near the $x$-axis in Figure 2, then the duration of the pulse is reduced from $0.22 T_{p}$ to $\sim T_{p}\left(\Delta r \perp / 2 r_{0}\right)^{2}$. The width is roughly the same for a patch $\Gamma^{-1}$ away from the $x$-axis as well. For example, assume that the arc responsible for the peak in Figure $4 a$ is only active for a small range of distances not near the $x$-axis. The dotted lines in Figure $4 a$ represent the possible peak shape if only a single patch is active during the $\delta$ function emission. The width of the patch can be adjusted to maintain a roughly constant peak width. If $\Delta T_{p}$ is the width of the peak at time $T_{p}$ in the time history, then, for the desired $\Delta T_{p}$ to be observed by the detector, $\Delta r_{\perp}$ must be limited to

$$
\Delta r_{\perp} \lesssim 2 c \Gamma\left(T_{p} \Delta T_{p}\right)^{1 / 2} .
$$

This predicts that $\Delta T_{p}$ should scale as $\Delta r_{\perp}^{2} / T_{p}$, whereas often peaks have the same width throughout the burst. Since there is no apparent reason why $\Delta r_{\perp}$ should scale as $T_{p}^{1 / 2}$, it seems unlikely that the patch size, alone, gives the temporal width of the peaks.

\subsubsection{Simultaneous Conditions and Burst Substructure}

The angular size, $\Delta r_{\perp}$, and the width of each of the emitting regions, $\Delta r_{\|}$, and the duration of emission $\Delta t$ are all constrained by the measured duration of the peaks, $\Delta T_{p}$. The upper limits of each of these quantities, $\Delta r_{\perp}, \Delta r_{\|}, \Delta t$ are

$$
\begin{aligned}
\Delta r_{\perp, \max } & =2 \Gamma c\left(T_{p} \Delta T_{p}\right)^{1 / 2}, \\
\Delta r_{\|, \max } & =c \Delta T_{p}, \\
\Delta t_{\max } & =2 \Gamma^{2} \Delta T_{p} .
\end{aligned}
$$

We define three quantities, $\Delta T_{\Delta r_{\perp}}, \Delta T_{\Delta r_{\|}}, \Delta T_{\Delta t}$, which are the components of the peak time $\Delta T_{p}$ contributed by $\Delta r_{\perp}, \Delta r_{\|}$, and $\Delta t$, respectively. Table 1 summarizes these quantities for each of the scenarios discussed. For a single shell that develops photon-emitting regions simultaneously, these three quantities are

$$
\begin{aligned}
\Delta T_{\Delta r_{\perp}} & =\frac{\Delta r_{\perp}^{2}}{4 \Gamma^{2} c^{2} T_{p}}, \\
\Delta T_{\Delta r_{\|}} & =\Delta r_{\|} / c, \\
\Delta T_{\Delta t} & =\Delta t / 2 \Gamma^{2} .
\end{aligned}
$$

From equation (16), it is evident that $\Delta r_{\perp}$ must be substantially larger than $\Delta r_{\|}$for the curvature of the expanding shell to have a significant effect on the time histories. If the radius of emission were large (for example, $T_{0} \sim 100 \mathrm{~s}$ ), the patch spanned by $\Delta r_{\perp}=2$ light-seconds would be essentially a planar region causing a $\delta$-function emission in the detector. A $\Delta r_{\|}$of 2 light-seconds, however, will cause a peak $2 \mathrm{~s}$ long in the detector.

\subsubsection{Simultaneous Conditions and Burst Duration}

The scaling of peaks within a burst does not generalize to the scaling of the complete burst time histories. Figure 5 shows an example of a mapping between the times for emission for various patches in the detector rest frame and their arrival at the detector. The gamma-ray arrival at the detector is sensitive to the angular offset from the line of sight at which the patch develops. The dashed lines represent the actual radii at which the patches reside. The solid lines

TABLE 1

Contributions to the Arrival Time of Photons from Duration of Emission and ThickNeSS OF EMITTING Regions IN VARIOUS ScENARIOS

\begin{tabular}{lccc}
\hline \hline Parameter & Patches & Seed Growth & Ambient Objects \\
\hline$\Delta T_{\Delta t} \ldots \ldots$. & $\frac{\Delta t}{2 \Gamma^{2}}$ & $\frac{\Delta t}{2 \Gamma^{2}}$ & $\frac{\Delta r_{\text {amb }}}{2 \Gamma^{2} c}$ \\
$\Delta T_{\Delta r_{\|}} \ldots \ldots$ & $\frac{\Delta r_{\|}}{c}$ & $\frac{c_{s}^{\prime} \Delta t}{c \Gamma^{2}}$ & $\frac{\min \left(\Delta r_{\|}, \Delta r_{\mathrm{amb}}\right)}{c}$ \\
$\Delta T_{\Delta r_{\perp} \ldots \ldots}$ & $\frac{\Delta r_{\perp}^{2}}{4 \Gamma^{2} c^{2} T}$ & $\frac{c_{s}^{\prime} \Delta t^{2}}{4 \Gamma^{6} c^{2}\left(T+T_{0}\right)}$ & $\frac{\Delta r_{\text {amb }}^{2}}{4 \Gamma^{2} c^{2} T}$ \\
$\Delta T_{p} \ldots \ldots$. & $\max \left\{\frac{\Delta r_{\|}}{c}, \frac{\Delta t}{2 \Gamma^{2}}, \frac{\Delta r_{\perp}^{2}}{4 \Gamma^{2} c^{2} T}\right\}$ & $\frac{\Delta t}{\Gamma^{2}}\left[\left(\frac{1}{2}\right)^{2}+\left(\frac{c_{s}^{\prime}}{c}\right)^{2}\right]^{1 / 2}$ & $\Delta T_{\Delta r_{\perp}}$ or $\Delta T_{\Delta r_{\|}}$a \\
\hline
\end{tabular}

${ }^{\text {a }}$ Depends on if object is collapsible or not; see text. 

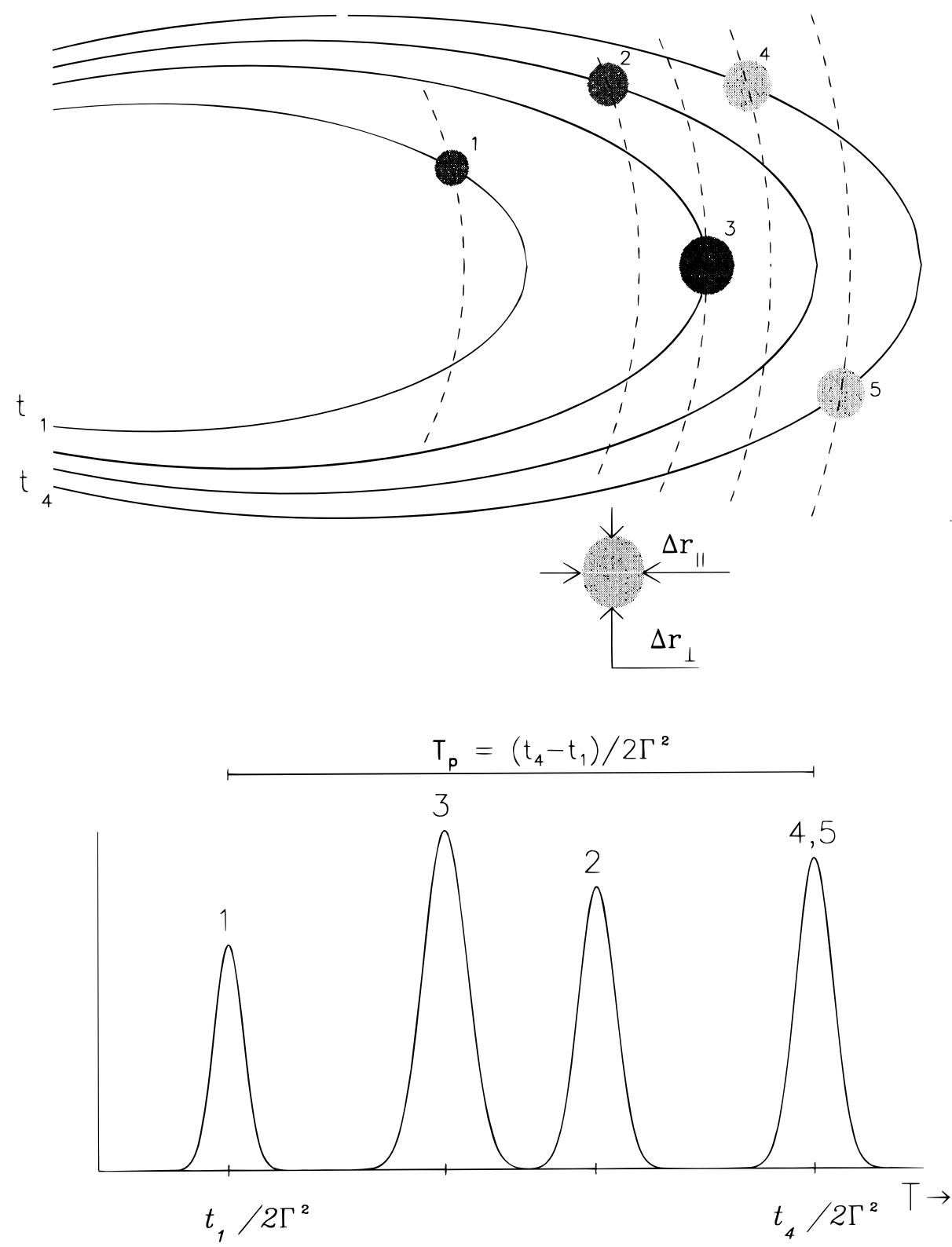

FIG. 5.-The relationship between observed time structure and the structure of the emitting region for the single, thin-shell model of the relativistic expansion. In the top panel, the elliptical curves are the surfaces that produce gamma rays that are seen at the same time as the detector. Five gamma-rayemitting regions produce peaks. The width of the individual peaks is related to the size of the individual emitting regions. The overall duration of the event is related to the overall size of the expansion.

represent the locus of points from which emitted photons arrive at the observer at the same time. Any difference in distance along the line-of-sight path, $\Delta d_{\text {los }}$, that the two photons are emitted in the rest frame of the detector requires a time $\Delta d_{1 \mathrm{os}} / c$ to travel. Thus, even a small distance off axis for a location of a patch can have a great effect on the time history.

Compare the photons emitted from site 2 to those from site 3. Site 2 photons were emitted at a smaller radius and thus earlier than site 3 , but they will arrive later because of their angular offset. Likewise, those emitted from site 4 and site 5 will arrive at the observer at the same time, even though site 4 photons were emitted significantly earlier. The degree by which the peaks may be scrambled can be seen in Figure 2. The region of the ellipsoid that is far from the line of sight corresponds to photons that were emitted at $T / 2$ earlier than photons emitted along the line of sight. Thus, the order of the peaks can be scrambled by $\sim T / 2$.

The order of arrival of the peaks is a function of both the time of emission and angular offset, and it is not possible to solve this inverse problem of peak order in the CMF (or the DRF) with certainty.

One conclusion that may be drawn from this analysis is that a shell cannot decelerate significantly during photon emission, otherwise a noticeable evolution in the time history would occur. In Figure 1 , the $\Delta T_{p}$ values in BATSE burst 2856 remain relatively constant throughout the burst. Since $T_{p} \propto \Delta t / \Gamma^{2}$, either there is a negative correlation between $\Delta t$ and $\Gamma^{2}$ or $\Delta t$ and $\Gamma^{2}$ do not change significantly throughout the burst. If the widths of peaks in any given GRB do not change by more than a factor of 3 or $4, \Gamma$ must not change by more than a factor of 2 within a given burst. 
Norris et al. (1996) reports that peaks vary only from $10 \mathrm{~ms}$ to $2 \mathrm{~s}$ in bright bursts, implying that $\Gamma$ cannot change by more than $10^{5 / 2}$ from burst to burst in all scenarios in which $\Delta t \propto \Gamma^{-2}$.

\subsection{Perturbation Growth}

In this second scenario, a gamma-emitting region grows from a seed in the CMF at speed $c_{s}^{\prime}$. In contrast to $\S 3.2$, the source size is limited by causality because the perturbation grows at a finite signal speed. Woods \& Loeb (1995) discuss such growth, although only on the surface of the shell rather than in three dimensions within the shell. First we examine seed growth in the context of forming the substructure of a burst, and then we consider how multiple seeds can form the complete time history of a GRB.

\subsubsection{Perturbation Growth and Burst Substructure}

Suppose that a perturbation grows at a speed $c_{s}^{\prime}$ for a time $\Delta t^{\prime}$ in the comoving frame. After a time $\Delta t^{\prime}$, the perturbation has grown in three dimensions to the size

$$
\Delta r_{\perp}^{\prime} \sim \Delta r_{\|}^{\prime} \sim c_{s}^{\prime} \Delta t^{\prime} .
$$

The gamma rays from this region will be emitted over both a range of times, spanning $\Delta t^{\prime}$, and a range of radii, $\Delta r_{\|}^{\prime}$, along the line of sight toward the detector. We look at how these effects couple to determine the mapping from the DRF to the detector's arrival time.

The duration of the seed growth will map in the same manner as duration has mapped in all situations (see Fig. 3 and $\S 2.1)$ :

$$
\Delta T_{\Delta t}=\frac{1}{2 \Gamma^{2}} \Delta t=\frac{1}{2 \Gamma} \Delta t^{\prime} .
$$

The thickness of the perturbation in the DRF will determine the time difference between two photons emitted at the same time in the DRF at the front and back of the shell (see Fig. 3). The maximum thickness of a patch that grows for a comoving time $\Delta t^{\prime}$ at speed $c_{s}^{\prime}$ is

$$
\Delta r_{\|}=\Delta r_{\|}^{\prime} \Gamma^{-1}=c_{s}^{\prime} \Delta t^{\prime} \Gamma^{-1}=c_{s}^{\prime} \Delta t \Gamma^{-2} .
$$

Thus, the maximum time spread due to thickness is

$$
\Delta T_{\Delta r_{\|}}=\frac{\Delta r_{\|}}{c}=\frac{c_{s}^{\prime}}{c} \Delta t \Gamma^{-2} .
$$

In this scenario, $\Delta r_{\perp}^{\prime} \sim \Delta r_{\|}^{\prime}$, which leads to

$$
\Delta T_{\Delta r_{\perp}}=\frac{c_{s}^{\prime} \Delta t^{2}}{4 \Gamma^{6} c^{2} T_{p}},
$$

which we can neglect.

We assume that the timescales from $\Delta T_{\Delta r \|}$ and $\Delta T_{\Delta t}$ add in quadrature to produce the observed peak:

$$
\Delta T_{p}=\frac{\Delta t}{\Gamma^{2}}\left[(1 / 2)^{2}+\left(c_{s}^{\prime} / c\right)^{2}\right]^{1 / 2} .
$$

\subsubsection{Perturbation Growth and Burst Duration}

The perturbations occurring in different locations on the expanding shell emit peaks whose order of arrival at the detector is affected by the angular offset from the line of sight to the detector in much the same way as those from simultaneous patches (see § 3.1.2). Again, this offset can scramble the arrival of the peaks by as much as $T_{p} / 2$, in which case any time evolution of the morphology of the peaks of photons emitted would still be apparent, although more difficult to recognize.

\subsection{Ambient Objects}

In the previous sections, the time structure arose from variations within the expanding shell. In this section, it is assumed that the time variation occurs because the shell interacts with inhomogeneities within the ambient medium. The shells might encounter clouds with variations in density (Rees \& Mészáros 1994). Or the shell might sweep over a source of photons, upscattering these ambient photons to gamma rays. For example, Epstein et al. (1993) pointed out that a typical GRB spectrum looks like an AGN spectrum that has been boosted. Furthermore, an AGN is one of the few places that has enough photons to flood the rest of the universe. Time variations might arise because the variations in the brightness of blobs ejected from the AGN (Epstein et al. 1993). A key problem with this suggestion is that welllocalized GRBs do not seem to be correlated with the luminous mass (the "no host" problem; Fenimore et al. 1993b). Another suggestion is that the bursts occur in the collapsed cores of globular clusters at cosmological distances and each peak is due to the shell running over an individual star (Shemi 1994; Shaviv \& Dar 1995). This scenario has difficulty explaining the FRED-like bursts and bursts with a large number of peaks.

\subsubsection{Ambient Objects and Burst Substructure}

Let us call the thickness of the expanding shell $\Delta r_{\|}$and the radius of the ambient object in the rest frame $\Delta r_{\text {amb }}$. Whether or not the ambient object collapses onto the shell in a key distinction that must be made in order to understand how the ambient object determines the time structure. For example, a shell interacting with a cloud might be expected to sweep all the material, effectively collapsing the cloud onto the surface of the shell. In this case, the photon production is similar to that of photons " $A$ " and " $a$ " in Figure 3. The shell keeps up with the photons it produces, and the duration of the collapse appears in arrival time as

$$
\Delta T_{\Delta t}=\frac{\Delta r_{\mathrm{amb}}}{2 \Gamma^{2} \mathrm{c}} .
$$

The ambient object is most likely symmetric, so the size in the perpendicular direction is the same as in the parallel direction. Thus, there will also be a contribution

$$
\Delta T_{\Delta r_{\perp}}=\frac{\Delta r_{\mathrm{amb}}^{2}}{4 \Gamma^{2} c^{2} T_{p}} .
$$

Since $\Delta r_{\|}$is zero for collapsible objects (by definition), the observed peak width will be some combination of $\Delta T_{\Delta T}$ and $\Delta T_{\Delta r \|}$. Unless $T_{p} \gtrsim \Gamma^{2} \Delta T_{p}$ (unlikely), the observed width will be dominated by $\Delta T_{\Delta r \|}$ and $\Delta r_{\text {amb }} \lesssim 2 \Gamma c\left(T_{p} \Delta T_{p}\right)^{1 / 2}$. The observed widths of peaks often do not scale as $T_{p}^{-1}$; thus, it seems that collapsible objects would have a difficult time explaining the time history.

The alternative is that the ambient source does not collapse but produces gamma rays on a scale of $\Delta r_{\mathrm{amb}}$. For example, stars would not collapse. In this case, the effect of $\Delta r_{\text {amb }}$ scales as a thickness much like photons "A" and "B" in Figure 3, and the width is determined from the light travel time across the overlap of the shell thickness and the ambient source thickness. Both $\Delta T_{\Delta T}$ and $\Delta T_{\Delta r_{\perp}}$ are small, 
SO

$$
\Delta T_{p} \sim \Delta T_{\Delta r_{\|}}=\frac{\min \left(\Delta r_{\|}, \Delta r_{\mathrm{amb}}\right)}{c} .
$$

We note that usually all the peaks appear to be about the same size (see Figs. $1 c$ and $7 c$ ). Stars might have similar sizes, but there might be an insufficient density of stars to account for the time history (see below). If the width of the peaks were due to the distribution of cloud sizes, one might expect a power-law distribution of peak widths, which is not observed. Thus, it appears that $\Delta r_{\|}$is smaller than $\Delta r_{\mathrm{amb}}$ and determines $\Delta T_{p}$. An upper limit on $\Delta r_{\text {amb }}$ is set by the condition that $\Delta T_{\Delta r_{\perp}}$ does not dominate. Therefore,

$$
\begin{aligned}
3 \times 10^{10} \mathrm{~cm} & =c \Delta T_{p}<\Delta r_{\mathrm{amb}}<2 \Gamma c\left(\Delta T_{p} T_{p}\right)^{1 / 2} \\
& \sim 3 \times 10^{13} \Gamma_{2} \mathrm{~cm},
\end{aligned}
$$

where $\Gamma_{2}$ is $10^{-2} \Gamma$.

\subsubsection{Ambient Objects and Burst Duration}

The duration of events defined by ambient objects depends on their distribution. The ambient objects responsible for the peaks are contained in the volume swept out of the shell within $\theta \sim \Gamma^{-1}$. This volume is a cone with a height of $2 \Gamma^{2} c T_{p}$ and a base radius of $\Gamma c T_{p}$. The volume grows as $T_{p}^{3}$, so one might expect the number of peaks per unit time to grow as $T^{2}$ (which, of course, is not observed). The shell apparently cannot utilize the ambient objects until after some time $T_{0}$, perhaps because some ISM material must first be swept up before the shell can interact with the ambient objects to generate gamma rays. If the burst duration is $T_{b}$ and $N_{b}$ peaks are observed, the required density is

$$
\rho_{\mathrm{amb}} \sim \frac{N_{b}}{(2 / 3) \pi \Gamma^{4} c^{3}\left[\left(T_{0}-T_{b}\right)^{3}-T_{0}^{3}\right]} .
$$

We have clear limits on $T_{0}: T_{0}$ cannot be much smaller than $T_{b}$ because we usually do not see the number of peaks increase as $T^{2}$. Furthermore, $T_{0}$ cannot be much larger than $T_{b} / 0.22$, since otherwise by local spherical symmetry, the objects at angles $\sim \Gamma^{-1}$ would produce peaks later than observed. Using $T_{0} \sim(5 / 2) T_{b}$,

$$
\rho \sim 1.4 \times 10^{8} N_{b} \Gamma_{2}^{-4}\left(100 / T_{b}\right)^{3} \mathrm{pc}^{-3} .
$$

Shaviv \& Dar (1995) imply that globular clusters might have a high enough stellar density to explain many bursts. However, the Shaviv \& Dar (1995) simulations were valid for instruments that make bolometric observations and, therefore, could observe many stars at angles larger than $\Gamma^{-1}$ despite the Lorentz shift in their energies. In the case of a shell moving through a globular cluster, $T_{0}$ should be $\sim 0$ in equations (27) and (11). The required density is larger than estimated by Shaviv \& Dar but still might be possible. However, equation (11) would give the average burst envelope and there seems to be no globular cluster geometry that would produce a FRED if $T_{0} \sim 0$.

We have treated $\Gamma$ as a free parameter. Often, one estimates that the photon-quiet phase is roughly the time is takes is energized the swept-up ISM, that is (Mészáros \& Rees 1993),

$$
E_{0} \sim \frac{4 \pi}{3} r_{0}^{3} \rho_{\mathrm{ISM}} m_{p} c^{2} \Gamma^{2}
$$

where $E_{0}$ is the total radiated energy, $\rho_{\text {ISM }}$ is the density of the ISM (typically $1 \mathrm{~cm}^{-3}$ ), and $m_{p}$ is the mass of a photon. We have shown that, due to shell symmetry, the radius of the photon-quiet phase is roughly $r_{0} \sim 2 \Gamma^{2} c T_{1 / 2} / 0.22$, where $T_{1 / 2}$ is the FWHM. Therefore,

$$
E_{0} \sim 1.3 \times 10^{32} T_{1 / 2}^{3} \Gamma^{8} .
$$

A reasonable value of $E_{0}$ is $10^{51}$ ergs. Certainly, $\Gamma \sim 10^{3}$ is unlikely, since it would require $E_{0} \sim 10^{58} \mathrm{ergs}$ unless $\rho_{\text {ISM }}$ is very small as it might be in a globular cluster (Shaviv \& Dar 1995). Thus, $\Gamma$ must be the order of $50-100$. This justifies the scaling of $\Gamma$ in equation (28).

Since $T_{p} \sim T_{0}+T_{b}$, the size of the region is $\sim 2 \Gamma^{2}\left(T_{0}\right.$ $\left.+T_{b}\right) \sim 0.07 \Gamma_{2}^{2}\left(T_{b} / 100\right)$ pc. For burst $2856, N_{b} \gtrsim 75$ and $T_{b} \sim 150 \mathrm{~s}$, so $\rho$ must be the order of $3 \times 10^{9} \mathrm{pc}^{-3}$ within a radius of $0.1 \mathrm{pc}$ if $\Gamma=100$.

\subsection{Thick Shell with Substructure}

Consider the situation in which material is ejected from the central site with a range of Lorentz factors. If the velocities of the emitted particles vary during the release time, a rough differentiation of velocities will occur and the expanding shell will spread to a finite thickness (Rees \& Mészáros 1994; Piran 1994). Suppose that the front of the shell moves at $\Gamma_{\max }$ and the back at $\Gamma_{\min }$. Let $T_{b}$ be the duration of the event as measured in the arrival time. Once the shell reaches a thickness of $\sim c T_{b}$, the time history can be formed simply by varying the emission of photons in spatial coordinates in the DRF; emission from a region with $\Delta r_{\|}=c T_{b}$ will map to a time history of duration $T_{b}$ in the detector (see Fig. 6). Each subpeak arises from a region the order of $c \Delta T_{p}$. Alternatively, a central site emitting for a duration $T_{b}$ could produce a thick shell with substructure even without a spread in $\Gamma$ (Piran 1994).

To be relevant, the thick shell must grow to a $\Delta r_{\|}=c T_{b}$ within a radius $r_{\max }=2 \Gamma_{\max }^{2} c\left(T_{0}+T_{b}\right)$. Otherwise, the duration is dominated by the shell curvature (i.e., $T_{b} \sim$ $0.44 T_{0}$ ). In Figure 6 , the vertical dashed line subtends $\sim 2 \Gamma^{-1}$. Let $d_{c}$ be the curvature within $\sim 2 \Gamma^{-1}$. If the shell is much thicker than $d_{c}$, the observed time structure can arise from spatial substructure within the thick shell. To be thick enough, $\Gamma_{\max }$ and $\Gamma_{\min }$ are related by

$$
\begin{aligned}
& \left(v_{\max }-v_{\min }\right) 2 \Gamma_{\max }^{2}\left(T_{0}+T_{b}\right) \\
& =\left(\frac{\Gamma_{\max }^{2}}{\Gamma_{\min }^{2}}-1\right) c\left(T_{0}+T_{b}\right)>0.44\left(T_{0}+T_{b}\right),
\end{aligned}
$$

which leads to

$$
\Gamma_{\max }>\sqrt{1.4} \Gamma_{\min }
$$

Rees \& Mészáros (1994) discuss shocks with a $\Gamma_{\max } / \Gamma_{\min }=$ 2 in which shells of varying $\Gamma$ values collide and form shocks that emit gamma rays. In that model, some slowspeed material leaves the central site first such that some high-speed material catches up and causes shocks. In our scenario, we emphasize that the high-speed material leaves first and stretches out the thickness of the shell. This effectively converts the shell into a parallel slab, and most of the problems associated with local spherical symmetry are eliminated.

\subsection{Central Engine}

Unlike the previous scenarios, a pure central engine does not involve a single, expanding shell that moves away from 

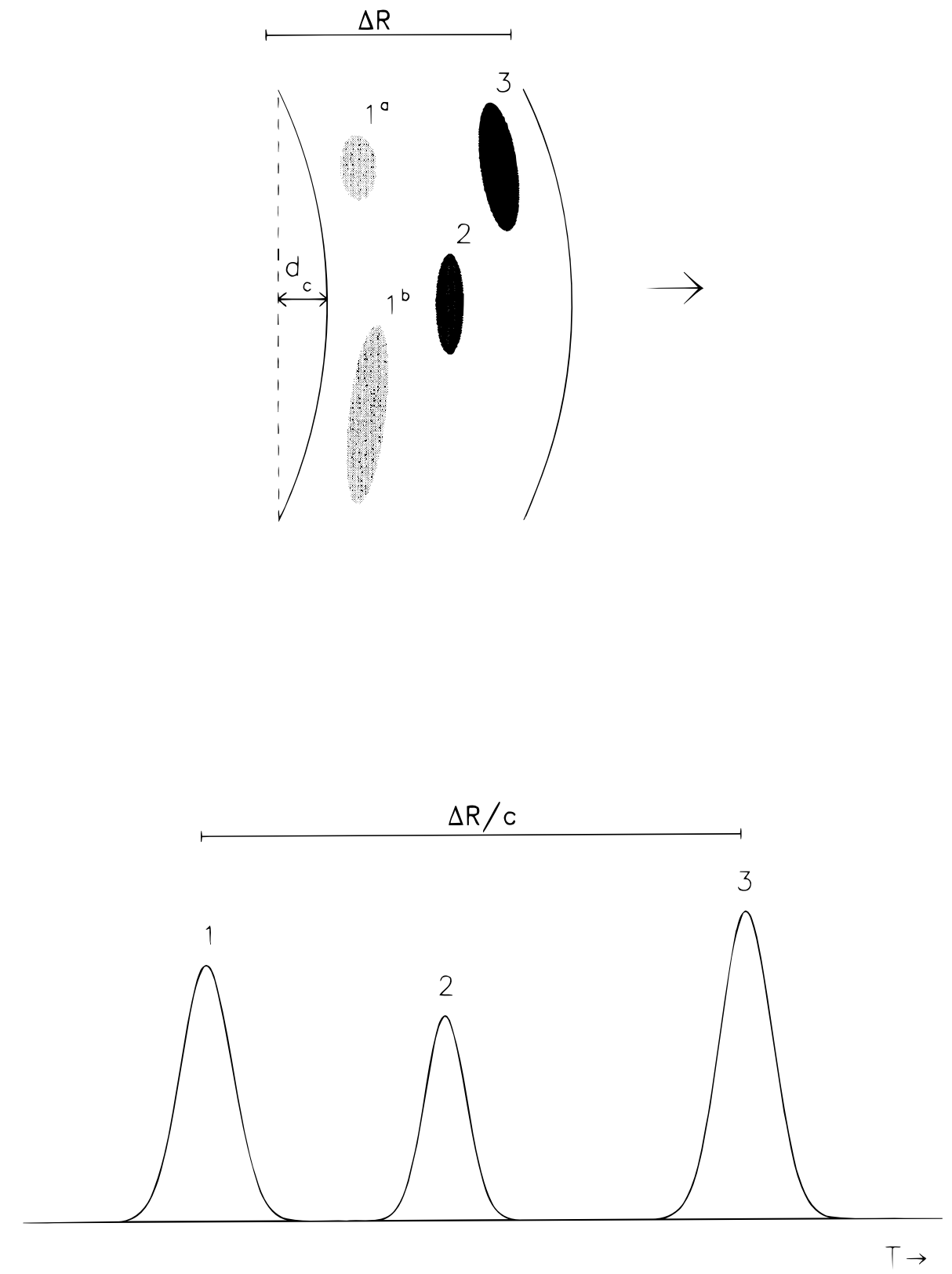

FIG. 6.-The relationship between observed time structure and the structure of the emitting region for a shell that has grown to be very thick and develops substructure within the shell. If the thickness $\left(\Delta R_{\text {parallel }}\right)$ is larger than the radius of curvature within $2 \Gamma^{2}$ (i.e., $\left.d_{c}\right)$, then the time structure will be dominated by the thickness and not local spherical symmetry. The duration of the event is determined from the thickness of the shell, and the individual peaks are determined from the subregions that develop into gamma-ray-producing areas.

the central site. Rather, the central engine emits multiple shells for a period roughly equal to the duration of the observed GRBs (up to $\sim 10^{3} \mathrm{~s}$ ). The shells expand to a radius of $\sim 2 \Gamma^{2} c \Delta T_{p}$. A release of these shells over a duration $T_{b}$ in the DRF (which is also the rest frame of the central site) results in the arrival of these shells in the detector over the same duration $T_{b}$. In this scenario, the radius of the shell does not exceed that given by the duration of the peaks in the time history.

\subsection{Summary of Relationships Between Temporal Variations and the Physical Size}

As the previous sections demonstrate, there are many ways to interpret the observed time histories. Table 2 summaries these. Here $\Delta T_{p}$ is a typical pulse width, $T_{b}$ is the observed duration of the event, $T_{0}$ is the duration of the photon quiet phase, and $T_{D}$ is the total duration of the event $\left(=T_{0}+T_{b}\right)$. In Table $2, R$ is the scale of the entire event and $\Delta R_{\perp}$ is the perpendicular scale of the emitting region. The $\Delta R_{\|}$is always less than $c \Delta T_{p}$, since thickness effects are not affected by the expansion (see $\S 2.1$ and Figure 3). A typical use of $R$ would be to estimate the amount of ISM material that the event sweeps up. A typical use of $\Delta R_{\perp}$ and $\Delta R_{\|}$is to estimate the photon density in calculations of photonphoton attenuation. For example, Fenimore et al. (1993a) explicitly assumed either a stationary photosphere or a central engine in estimating the size of the region relevant for calculating the photon-photon attenuation. Nayakshin $\&$ Fenimore (1996) use the results of this paper to obtain a more realistic estimate. 
TABLE 2

Size Estimates From Various Models ${ }^{\mathrm{a}}$

\begin{tabular}{|c|c|c|}
\hline Model & $R$ & $\Delta R_{\perp}$ \\
\hline 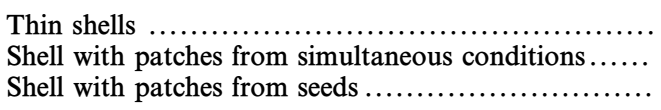 & $\begin{array}{l}2 c \Gamma^{2} T_{D} \\
2 c \Gamma^{2} T_{D} \\
2 c \Gamma^{2} T_{D}\end{array}$ & $\begin{array}{l}2 c \Gamma T_{D} \\
2 c \Gamma\left(T_{p} \Delta T_{p}\right)^{1 / 2} \\
\quad c \Gamma \Delta T_{p}\end{array}$ \\
\hline Thick shell from spread in $\Gamma$.. & $2 c T_{D}\left(\frac{1}{\Gamma_{\min }^{2}}-\frac{1}{\Gamma_{\max }^{2}}\right)^{-1}$ & $c T_{b}, c \Gamma \Delta T_{p}$ \\
\hline $\begin{array}{l}\text { Thick shell from central engine } \ldots \ldots \ldots \ldots \ldots \ldots \ldots \ldots \ldots \\
\text { Multiple shells from central engine } \ldots \ldots \ldots \ldots \ldots \ldots \ldots \ldots \\
\text { Stationary photosphere } \ldots \ldots \ldots \ldots \ldots \ldots \ldots \ldots \ldots \ldots \ldots \ldots\end{array}$ & $\begin{aligned} & >c T_{b} \\
2 c & \Gamma^{2} \Delta T_{p} \\
c & \Gamma \Delta T_{p}\end{aligned}$ & $\begin{array}{l}c T_{b}, c \Gamma \Delta T_{p} \\
2 c \Gamma \Delta T_{p} \\
\quad c \Delta T_{p}\end{array}$ \\
\hline
\end{tabular}

${ }^{\mathrm{a}} \Delta R_{\|}$must always be $<c \Delta T_{p}$.

In the first three cases in Table 2, $R$ arises from the size of the single shell (see eq. [3]). Patches from simultaneous conditions are limited by equation (14), whereas patches that are limited by causality (i.e., growth from a seed) are limited by equation (22). Thick shells with substructure are dominated by thickness effects, so the duration arises from the overall thickness of the shell, and the individual emitting regions are set by $\Delta T_{p}$. The thick shell with substructure could arise from either a single shell that spreads out due to a spread in $\Gamma$ (see $\S 3.4$ ) or by a central engine that feeds a shell continuously. In the latter case, $R$ is limited to be $c T_{b}$. Finally, for completeness, we include a stationary photosphere with a relativistic wind (see Paczyński 1986, 1990). Since there is no motion of the emitting surface, the size is limited by the light travel across the observable surface (i.e., the surface within $\Gamma^{-1}$ ).

Although at first glance, many of the scenarios that we have discussed seem to be very similar, Table 2 demonstrates that one obtains different estimates of the overall size or the size of the emitting region in each case. The overall size of the region can vary from $c T_{D}$ to $2 \Gamma^{2} T_{D}$, and the perpendicular size of the emitting region can vary from $c \Delta T_{p}$ to $2 c \Gamma T_{p}$. We have explicitly only considered kinematic limits and have not considered the fireball physics that might cause these changes. Several types of fireball models have been considered in the literature. Some involve "impulsive" fireballs in which the time structure arises in the expanding shell. In Table 2, the impulsive model include the thin shell, patches from simultaneous conditions (probably caused by ISM variations; see Mészáros \& Rees 1993), patches from seeds (probably caused by internal shocks; see Rees \& Mészáros 1994, Piran 1994; Woods \& Loeb 1995), and the multiple shell scenario. Other models involve "continuous energy input fireballs." In Table 2, these models include the thick shell scenarios (see Rees \& Mészáros 1994; Waxman \& Piran 1994) and the stationary photosphere (Paczyński 1990). The thick shell from a spread in $\Gamma$ is a hybrid in which the time structure might arise from the duration of the energy input or the spread in size after the impulsive release.

\section{DISCUSSION}

We have outlined five general scenarios in which most existing models of GRB emission can fall. Each of these scenarios has constraints outlined in Table 1 on photon emission based on the observations. We turn now to three general classifications covering the majority of burst types and discuss how the burst scenarios can be used to account for GRB time structure. The three classifications are bursts with a single or a few isolated sharp spikes, FREDs, and bursts with complex time structure.

\subsection{Sharp, Isolated Spikes}

The time histories of bursts that fall into this category consist of one or, at most, a few sharp spikes occurring within a short duration. A example of such a burst is shown in Figure $1 a$. Note that the fall time is roughly the same as the rise time, so this is not just a very narrow FRED. Such spikes can be examined by one of three scenarios. First, the emission may arise from a full shell with local spherical symmetry emitting photons at a low $t_{0}$ so that the FWHM due to the shell $\left[\sim 0.22 t_{0} /\left(2 \Gamma^{2}\right)\right]$ is smaller than the other contributors to the width. For example, consider the contribution arising from how the gamma-emitting region could grow from a seed over time $\Delta t$. The lack of a FRED-like shape in Figure $1 a$ implies that $t_{0} \lesssim \Delta r / 2$. Since $\Delta T_{p}$ of Figure $1 a$ is only $\sim 0.5 \mathrm{~s}, t_{0}$ must be $\lesssim 5 \Gamma^{2}$, or $5 \times 10^{4} \mathrm{~s}$ for $\Gamma=100$.

Second, the photons that cause the single spike could arise from a single localized patch or seed at a larger $t_{0}$. Although the bulk material might expand under local spherical symmetry, the conversion to gamma rays could be very asymmetric. In this case, $r_{0}$ is not limited to being small because $\Delta r_{\perp}$ is assumed to be small to produce the sharp time profiles observed. The region must, however, be significantly smaller than the total region that lies within the beam toward the detector. If the photons we observe were emitted from only a small portion of the shell, then the total energy requirements of the shell become greater by the ratio of the total surface to that of the emitting surface. We define $f$ to be the filling factor; that is, the ratio of the observed emission to which we would be expected under local spherical symmetry of the gamma-ray production:

$$
f \propto \frac{\int P(\theta, \phi, t) \Lambda^{-3} d A}{\int \Lambda^{-3} d A} .
$$

For a patch to give a timescale of only $\Delta T_{p}$, the filling factor needs to be

$$
f \propto \frac{r_{\perp}^{2}}{r^{2} \Gamma^{2}}=\frac{\Delta T_{p}}{T_{p}}=\frac{\Delta t_{p}}{t_{p}} .
$$

The shell would have produced a FRED if all of it emitted but only a small region (e.g., patch in Fig. 5) emitted resulting in the dotted line in Figure $4 a$. It is normally assumed that the fireball energy is converted first to kinetic energy of the shell and then converted to gamma rays. To explain narrow spikes if $t_{0}$ is larger than $\sim 10^{4}$, one must assume either that the material is confined to a pencil beam 
with an opening angle of only $\sim \Delta t_{p} / t_{0}$ or that only a region with a size $\sim \Delta t_{p} / t_{0}$ converted its energy into gamma rays. In the latter case, $f$ is very small, and most of the energy of the fireball is never converted into gamma rays. This could raise the required energy to larger than the available by merging neutron stars.

Third, a shell sweeping past a star (§ 3.3) can account for single sharp spikes. However, since the star interacts only with a small fraction of the shell's surface $\left(\sim \Delta r_{\mathrm{amb}} / \Gamma c t_{0}\right)$, the filling factor $(f)$ is, again, small.

\subsection{FREDs}

FREDs account for a significant proportion of all GRB time histories. They can be explained easily by a single, full shell emitting for a short $d t$ at a radius $r_{0}=2 \Gamma^{2} c T_{0}$, where $0.22 T_{0}=\Delta T_{p}$. This $\delta$-function emission discussed in $\S 2$ produces an envelope very similar to many observed FREDs. It has a sharp rise ending in a cusp and falls off slowly to background. No other scenarios can explain so simply the morphology of FRED GRBs. The FRED shape in arrival time is determined solely from $T_{p}$. For example, we have fitted equation (8) to the time history of burst 1885 (see Fig. $1 b$ ). The FWHM of the FRED is about $35 \mathrm{~s}$, so $T_{0}$ is $157 \mathrm{~s}$. We have placed $T=0$ in Figure $1 b$ about $157 \mathrm{~s}$ before the FRED. From equation (30), one can estimate $\Gamma$. Even using a large value of $E_{0}\left(10^{52} \mathrm{ergs}\right), T_{1 / 2}$ equal to $35 \mathrm{~s}$ implies that $\Gamma<80$.

Figure $7 a$ shows burst 678 , which has statistically significant subpeaks within an envelope that is roughly FREDlike. The $T_{1 / 2}$ is $7.7 \mathrm{~s}$, so equation (30) implies that $\Gamma \sim 140$. The presence of the subpeaks is explained easily as separate regions that became active roughly simultaneously in the DRF. If the conversion of bulk motion gamma rays has local spherical symmetry on average over the shell, some regions could be more efficient at producing gamma rays than others. The peaks in the tail of the FRED arrive later at the detector because they are due to regions offset from the line connecting the central explosion and the observer. Since the late-arriving peaks are due to regions offset by angle $\theta$ from the tip of the ellipsoid, those regions should have a different Lorentz boost factor. The region near the tip of the ellipsoid produces the signal near the peak of the FRED, and its spectrum is the comoving spectrum boosted by $\Lambda_{p}^{-1}=2 \Gamma$ (see eq. [1]). For a peak arriving at time $T$, the boost factor is $2 \Gamma T_{p} / T$. Thus, we predict that the relative boost factor in a FRED is simply $T_{p} / T$. Note that one needs to know where $T=0$ is, but that can be found from the shape of the FRED. There is a one-to-one mapping of the relative boost factor to the FRED shape; this is shown in Figure $4 b$. In Figure $7 a$, the arrow indicates where the spectrum should be boosted by a factor of 2 less than at the peak of the FRED (assuming that $\alpha=1.5$ ). Indeed, burst 678 and others FREDs show substantial softening through the tail. A detailed analysis to see if the softening is consistent with Figure $4 b$ is in preparation. Since time is also affected by the Lorentz transformation, the average temporal structure at the arrow should be twice as long as the temporal structure near the peak.

In contrast to burst 678, some GRBs (e.g., burst 451) have extremely smooth profiles in which the intensity follows a clear pattern. Smooth-profile GRBs could present special problems for relativistic shells. In the context of relativistic shells, the smoothness arises because of nearly identical conditions on a shell over distances the order of $\sim \Gamma c T$.
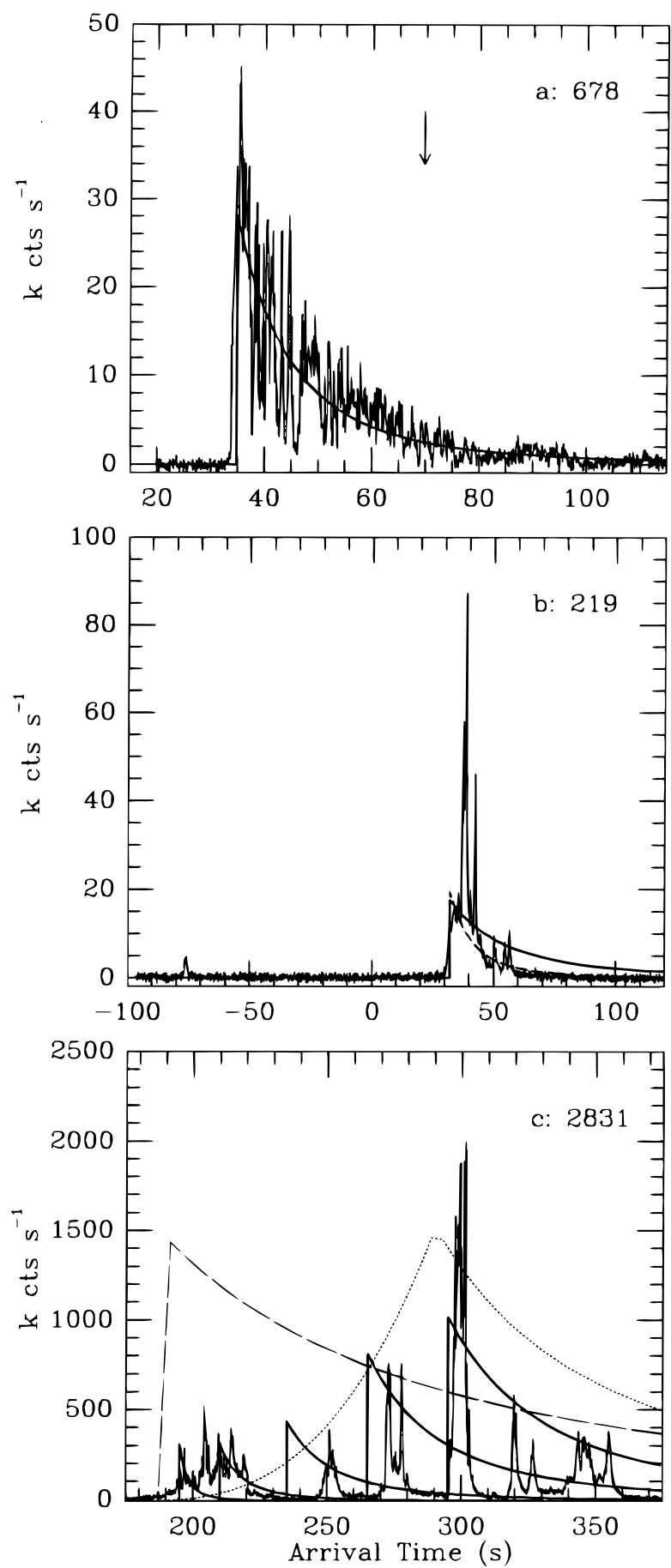

FIG. 7.-Fits of expected signals from relativistic shells of GRB time histories. (a) Burst 678 has a FRED-like envelope but many individual peaks. The solid curve is the expected signal if the shell started to expand at time 0 . The arrow points to the time at which the spectrum should be softer by a factor of 2 . The time structure should also be dilated by a factor of 2 . (b) The dashed line is a fit of a relativistic shell to Burst 219. The deduced start time for the shell is at time 0 . This burst had a precursor $100 \mathrm{~s}$ before this peak. The solid line is the expected shape, assuming that the shell started at the time of the precursor. Either only a very small amount of the shell was converted into gamma rays, or this is an example of a central engine; that is, multiple shells spread out over 100 s. (c) Burst 2831, a long complex burst. The dashed line is an expected shape from a single shell that becomes gamma active for a short time. The dotted curve is the expected shape for a shell that becomes active over a range of times. The five solid curves are the shapes expected if a single shell becomes active at five different times. In all cases, only a small fraction of the shell's surface generates the gamma rays. This demonstrates the "shell symmetry" problem: the envelopes of most GRB time histories are not consistent with local spherical symmetry. 
Causality is not violated because the coordinated brightness comes about from local spherical symmetry, not by the propagation of a faster than light signal. However, if the smoothness of the intensity is from nearly identical conditions, then the spectral softening should be expected to follow closely $\Lambda / \Lambda=T_{p} / T$. If it does not follow $\Lambda / \Lambda_{p}$, then the smoothness probably arises from the propagation of a signal, and the perpendicular size is limited by causality $\left(c \Gamma \Delta T_{p}\right)$ rather than by the shell size $\left(2 c \Gamma T_{D}\right.$; see Table 2$)$. In such a case, the filling factor is very small $\left(\sim\left[T_{p} / T_{D}\right]^{2}\right)$, and the gamma-ray-producing regions must subtend only $\sim\left(\Delta T_{p} / T_{D} / \Gamma\right)^{2}$ sr. Since the region is small, the required $\Gamma$ to avoid strong photon-photon attenuation is larger (Nayakshin \& Fenimore 1996). The net result is that the emission would be confined to angular scales of $<10^{-8} \mathrm{sr}$, a serious problem for any cosmological model. Thus, if the smooth-profile GRBs do not have the predicted softening pattern, the emitting region is probably unacceptably small.

Figure $7 b$ shows burst 219 , which has several large peaks on a FRED-like envelope. Thus, it is similar to burst 678 in Figure $7 a$, except that some patches appear to have a wider range of gamma-ray efficiency producing a wider variation in peak height. The dashed curve is the widest FRED-like shape that we could fit to the envelope of emission. Including more of the large peaks in the fit would make the FWHM of the FRED-like shape smaller. Based on the FWHM, the time equal to zero in Figure $7 b$ is when the shell responsible for the emission started. We believe that it is reasonable to attribute the peak shape to an expanding relativistic shell that became gamma active after $T_{0} \sim 30 \mathrm{~s}$. However, the peak shown in Figure $7 b$ is actually the second peak from this event. The first peak occurred $100 \mathrm{~s}$ earlier. If one assumes a single explosion (e.g., two merging neutron stars), $T_{p}$ must be at least $100 \mathrm{~s}$ for the second peak. The solid curve in Figure $7 b$ shows the expected FRED-like shape based on $T_{p}$ being at least $100 \mathrm{~s}$. Either the two peaks in burst 219 have a temporal separation because they were caused by two separate explosions (i.e., a central engine), or the second peak must have a low $f$, and only a small portion of the shell within $\Gamma^{-1}$ became gamma active. We have used $\zeta=0$ (see eq. [12]). If $\zeta>0$, the discrepancy between the observed envelope and that predicted from the precursor would be even larger.

We consider burst 219 to be an excellent example that points to central engines as the origin of the overall duration of GRBs. Koshut et al. (1995) define precursor activity as weak emission that is separated from the remaining emission by an interval that is at least as long as the remaining emission. Using the notation of Koshut et al. (1995), any burst that has $\tau_{\text {main }} \gtrsim 2.5 \Delta t_{\text {det }}$ is a strong contender for being a central engine. (Here one should use an estimate of the duration of the FRED-like component for $\tau_{\text {main }}$.) A few percent of the BATSE bursts probably satisfy the condition. A significant difficulty that lies in a central engine explanation is that cosmological objects must emit $\sim 10^{50}$ ergs on a timescale that compares to the observed durations.

\subsection{Complex Bursts}

This category includes all bursts whose time histories contain more substructure than those discussed above. The bursts in Figures $1 c$ and $7 c$ fall into this category. We will discuss three scenarios that could produce long complex bursts. The first is trivial: a central engine can explain any time history. The second involves expanding shells that convert their energy into gamma rays because of some process internal to the shell (such as shocks). The third scenario involves interactions with ambient objects external to the shell such as clouds or stars.

The basic problem with attempting to explain large complex scattering bursts with shells that expand and then convert their kinetic energy into gamma rays is that it should be possible to discern a FRED-like shape or a sum of FRED-like shapes. In Figure $7 c$ we have attempted to identify ways that $V(T)$ could account for the time history. A single shell (e.g., the dashed curve in Fig. 7c) implies low $f$ : many portions of the shell did not convert their energy into gamma rays. Multiple shells could help increase $f$. The five solid lines represent shells at five different $T_{p}$-values. Note that each successive shell produces a FRED-like structure that is progressively wider. Again, $f$ would be small. Another alternative is a shell that emits for a range of times. The dotted line in Figure $7 c$ is a fit using equation (11). Again, the quiet times have to be attributed to regions that did not turn on as gamma-ray sources, implying a low $f$.

The third scenario that might be applicable to long complex bursts is one in which the time structure comes from the shell interacting with ambient inhomogeneities such as clouds in the ISM or stars. Collapsible objects (see $\S 3.3$ ) should produce peaks that have variations in their widths (see $\Delta T \Delta r_{\perp}$ in Table 1). Long complex bursts often have peaks that are remarkably similar throughout the burst (see Fig. 1c). One expects $\Gamma$ to vary as the shell loses energy, and there is no reason why all the ambient objects should be the same size. Only peaks from noncollapsible objects are independent of $\Gamma$ and the size distribution of the objects. Ambient objects are acceptable only if the objects are noncollapsible, are between $3 \times 10^{10}$ and $3 \times 10^{13} \mathrm{~cm}$ in size eq. [26], and have density on the order of $3 \times 10^{9}(\Gamma /$ $100)^{-4} \mathrm{pc}^{-3}$ over a region of $\sim 0.1 \Gamma_{2}$ pc (eq. [28]). It is not clear what ambient objects can fulfill these characteristics. Stars have the right size and are noncollapsible but may not have the requisite density. ISM variations are probably collapsible, and they probably usually have larger sizes. Previously, it was thought that $T_{0}$ could be very large, reducing the required density. However, large $T_{0}$ cannot be much bigger than the observed duration, since otherwise additional peaks would be seen at $\sim 0.22 T_{0}$. Perhaps $\Gamma$ is as large as $10^{3}$. Then the required objects can be as large as $10^{14} \mathrm{~cm}$, the density as small as $3 \times 10^{6} \mathrm{pc}^{-3}$ within a radius of $10 \mathrm{pc}$. However, then equation (30) requires $E_{0}$ to be $10^{62}$ ergs. In any case, these ambient objects cannot be arranged randomly, since the separation in the peaks occurs with a log-normal distribution rather than with a random distribution (Li \& Fenimore 1996).

Thus, from the kinematics and the observed time structure, we conclude that it is very difficult to explain long complex bursts with a single shell (except for FREDs). It seems that such structure can arise only from either a central engine or a thick shell with substructure (e.g., Rees \& Mészáros 1994; Piran 1994; Waxman \& Firan 1994).

\section{CONCLUSIONS}

The Large Lorentz factor required to allow the highenergy photons escape from GRBs results in a shell that is only visible when seen head on; GRBs rarely appear as the sides of a jet. When viewed head on, the curvature of the shell is just as important as the expansion in determining the temporal structure. Thus, local spherical symmetry 
needs to be assumed. The principle of local spherical symmetry can explain easily the FRED-like bursts. We predict that, if FREDs are the result of a shell, they can be fitted by $T_{0}\left(T / T_{0}\right)^{-\alpha-2}$, where the only true free parameter is the start time. The spectra should soften as $T / T_{0}$. FRED-like bursts with precursors provide a strong argument that the time histories are due to a central engine rather than a single release of energy (see Fig. 7b).

If smooth-profile bursts do not soften as $T / T_{0}$, then the size of the emitting region is probably determined by lighttravel time and, therefore, extremely small: less than $\left(\Delta T_{p} / T_{D} / \Gamma\right)^{2} \sim 10^{-8}$ sr.

Short spikes must have very small photon-quiet phases $\left(10^{4} \mathrm{~s}\right)$, or the material is beamed on a scale much smaller than $\Gamma^{-1}$, or the conversion of bulk motion to gamma rays must have a small filling factor; that is, only a small portion of the shell is ever induced into producing gamma rays. This would raise the overall energy requirements.

Long complex bursts present a myriad of problems for the models. The duration of the event is $\sim t_{0}\left(2 \Gamma^{2}\right)$. The long duration cannot be due to large $t_{0}$, since it requires too much energy to sweep up the ISM (eq. [30]). Nor can it be due to small $\Gamma$ if the time variation is due to ambient objects, since the density of such objects is unreasonable (eq. [28]). Long events with small $\Gamma$ and time variations due to shocks must explain why they almost always violate local spherical symmetry. We introduce the "shell symmetry" problem for cosmological GRBs: models that arise from a single, central release of energy that forms a relativistic shell must somehow explain how either the material is confined to pencil beams narrower than $\Gamma^{-1}$ or how a shell can have a low filling factor with the resulting higher energy requirements. Without such explanations, local spherical symmetry requires a FRED-like shape. Ambient objects can be inhomogeneous, and there could be situations in which they would produce a low filling factor. However, this might help to explain a few non-FRED time histories, whereas most GRB time histories are non-FRED. We show that the thickness (in contrast to the duration) of the gamma-ray emission can dominate easily because the contribution from the thickness is not affected by $\Gamma$ (see Table 1). Many bursts have $1 \mathrm{~s}$ timescales, so the emitting region cannot be thickner than $1 \mathrm{~s}$, whereas the radius of the shell is $2 \Gamma^{2} T$, where $T$ can be hundreds of seconds. The number of peaks per unit time does not usually increase as $T^{2}$; thus, $T_{0}$ must be large. However, a large $T_{0}$ should produce a FRED-like envelope with a width of $\sim 0.22 T_{0}$. The width of the peaks can remain remarkably constant throughout a long burst. Only "noncollapsible" ambient objects produces peak widths independent of $\Gamma$ and the size distribution of the ambient objects because noncollapsible objects produce peak widths by thickness effects rather than duration effects. Explanations that depend on duration (e.g., growth of shocks) have a pulse width that depends on $\Delta t / \Gamma^{2}$ (see Table
1). Thus, a constant peak width requires that either $\Gamma$ changes in time as $\Delta t^{1 / 2}$ (no theory seems to predict this), or that $\Gamma$ and $\Delta t$ remain constant through the photon emission. Although it is reasonable that $\Delta t$ is constant, most theories expect $\Gamma$ to decrease as the shell loses energy.

Most of the above problems arise because the local spherical symmetry requires weaker emission from material at $\theta \sim \Gamma^{-1}$, which will arrive later by $\sim 0.2 T_{0}$. The "thick shell with substructure" model (\$3.4) is a promising way to overcome this and still have a single release of energy at the central site. If the shell thickens such that it is much wider than the radius of curvature of the shell within $\Gamma^{-1}$, it will act as a parallel slab, not a spherical surface. A parallel slab with embedded, small substructure that grows from a seed (as shocks might) could explain most GRB time history envelopes.

Table 2 summarizes various models and what sizes one obtains based on the observed $T_{D}$ and $\Delta T_{p}$. The overall size of the region can vary from $c T_{D}$ to $2 \Gamma^{2} T_{D}$, and the perpendicular size of the emitting region can vary from $c \Delta T_{p}$ to $2 c \Gamma T_{p}$.

Merging neutron stars release their energy over a short period of time and require an expanding shell to explain the observed time variations. The expansion can be relativistic to allow the escape of photons well above the pair production threshold. Our analysis indicates that for burst time histories, a central engine explanation is preferred in which the duration is dictated by the duration of the energy release at the central site. Failed supernova (Woolsey 1993; Hartmann \& Woolsey 1995) at cosmological distances might provide the central engine. However, failed supernova do not produce the superrelativistic shells necessary to explain the high-energy emission. Alternatively, neutron stars in the halo of our Galaxy could provide the central site, since the energy release within any peak is not large enough to destroy the object. The requisite relativistic expansion is smaller at distances commensurate with halo models can be produced with a much smaller $\Gamma$ (see Nayakshin \& Fenimore 1996).

If GRBs are central engines, then each peak is probably due to a relativistic expanding shell. In that case, the results of this paper can be applied to the individual peaks. Most peaks have faster rises than falls (Norris et al. 1996, and equation (11) could be used to estimate their characteristics. The rise of the pulse is related to the duration of the photonactive phase, and the fall is related to the duration of the photon-quiet phase.

This work was done under the auspices of the US Department of Energy and was funded in part by the Gamma Ray Observatory Guest Investigator Program. We thank C. Dermer, H. Li, C. Ho, and P. Mészáros for useful suggestions and corrections.

\section{REFERENCES}

Epstein, R. I., Fenimore, E. E., Leonard, P. J. T., \& Link, B. 1993, Ann. NY Acad. Sci., 688, 565

Fenimore, E. E. Epstein, R. I. \& Ho, C. 1993a, A\&AS, 97, 59

Fenimore, E. E., et al. 1992, in Gamma-Ray Bursts: Huntsville, 1991, ed W. S. Paciesas \& G. J. Fishman (New York: AIP), 158 1993b, Nature, 366, 40

Hartmann, D. H., \& Woolsey, S. E. 1995, Adv. Spac. Res., 15(5), 143

Hurley, K, et al. 1995, Nature, 372, 652

Katz, J. I. 1994, ApJ, 422, 248

Koshut, T. M., et al. 1995, ApJ, 452, 145

Krolik, J. H., \& Pier, E. A. 1991, ApJ, 373, 227

Lamb, D. Q. 1995, PASP, 107, 1152

Li, H., \& Fenimore, E. E. 1996, ApJ, 469, L115

Meegan, C. A., Fishman, G. J., Wilson, R. B., Paciesas, W. S., Brock, M. N,

Horack, J. M., Pendleton, G. N., \& Kouveliotou, C. 1992, Nature, 335 , 143

Mészáros, P., \& Rees, M. J. 1992, MNRAS, 258, 41P

.1993, ApJ, 405, 278

1994, MNRAS, 269, L41

Nayakshin, S., \& Fenimore, E. E. 1996, in preparation

Norris, J. P., et al. 1996, ApJ, 459, 393

Paczyński, B. 1986, ApJ, 308, L43 
Paczyński, B. 1990, ApJ, 363, 218

1995, PASP, 107,1176

Piran, T. 1994, in Gamma-Ray Bursts: Huntsville, 20-22 October, 1993 ed. G. J. Fishman, J. J. Brainerd, \& K. C. Hurley (New York: AIP), 495

Piran, T., \& Shemi, A. 1993, ApJ, 403, L67

Piran, T. Shemi, A., \& Narayan, R. 1993, MNRAS, 263, 861

Rees, M. J. 1966, Nature, 211, 468

Rees, M. J., \& Mészáros, P. 1994, ApJ, 430, L93
Sari, R., \& Piran, T. 1995, ApJ, 455, L143

Schmidt, W. K. H. 1978, Nature, 271, 525

Shaviv, N., \& Dar, A. 1995, MNRAS, 277, 287

Shemi, A. 1994, MNRAS, 269, 1112

Waxman, E \& Piran, T. 1994, ApJ, 433, L85

Woods, E., \& Loeb, A. 1995, ApJ, 453, 583

Woolsey, S. E. 1993, ApJ, 405, 273 